\title{
Ubiquitin Conjugation of Hepatitis B Virus Core Antigen DNA Vaccine Leads to Enhanced Cell-Mediated Immune Response in BALB/c Mice
}

\author{
Jian-Hua Chen ${ }^{1}$, Yong-Sheng Yu ${ }^{1}$, Hong-Hong Liu ${ }^{1}$, Xiao-Hua Chen ${ }^{1}$, Min Xi ${ }^{1}$, Guo-Qing \\ Zang $^{1}$, Zheng-Hao Tang $^{1^{*}}$ \\ ${ }^{1}$ Department of Infectious Diseases, Sixth People's Hospital Affiliated to Shanghai Jiaotong University, Shanghai, China
}

\begin{tabular}{l}
\hline A R T I C L E I N F O \\
\hline Article Type: \\
Original Article \\
\hline Article history: \\
Received: 24 Feb 2011 \\
Revised: 21 Mar 2011 \\
Accepted: 01 Apr 2011 \\
\hline
\end{tabular}

Keywords:

DNA vaccine

Ubiquitin

Hepatitis B core antigen

\section{A B S T R A C T}

Background: Nearly 350 million persons worldwide are chronically infected with hepatitis B virus (HBV). Ubiquitin (Ub) is a highly conserved small regulatory protein, ubiquitous in eukaryotes, that usually serves as a signal for the target protein that is recognised and degraded in proteasomes. The Ub-mediated processing of antigens is rapid and efficient and stimulates cell-mediated immune responses. Accordingly, Ubmediated processing of antigens has been widely used in chronic-infection and cancer studies to improve immune response.

Objectives: Many clinical trials have shown that DNA vaccine potency needs to be greatly enhanced. Here, we report a new strategy for designing an HBV DNA vaccine using the ubiquitin (Ub) sequence. The aim of this study was to investigate a novel DNA vaccination, based on the expression of HBV core antigen ( $\mathrm{HBcAg}$ ), fused to Ub to enhance DNA vaccine potency.

Materials and Methods: Mouse ubiquitin fused to the HBcAg gene and cloned into the eukaryotic vector pcDNA3.1 (-). BALB/c mice were immunized with recombinant pUbHBcAg or pHBcAg DNA vaccine. Lymphocyte proliferation assay, intracellular IFN- $\gamma$ assay, CTL cytotoxicity assay, and antibody assay were performed to analyze the cellular and humoral immune responses to our DNA constructs.

Results: HBcAg was expressed effectively in the COS-7 cells that were transiently transfected with pUb-HBcAg. Strong anti-HBc IgG responses were elicited in mice that were immunized with pUb-HBcAg. The endpoint titers of anti-HBc peaked at 1:656100 on the 42nd day after the third immunization. pUb-HBcAg stimulated greater lymphocyte proliferation and induced higher levels of IL-2 and IFN- $\gamma$ and a greater percentage of HBcAg-specific CD8+ T cells in mice than pHBcAg. In the CTL assay, the specific lysis rate reached $56.5 \%$ at an effector:target ratio of 50:1 in mice that were immunized with pUb-HBcAg.

Conclusions: pUb-HBcAg elicits specific anti-HBc responses and induces HBc-specific CTL responses in immunized BALB/c mice. Our results imply that Ub can be used as a molecular adjuvant that enhances the potency of DNA vaccines.

๑ 2011 Kowsar M.P.Co. All rights reserved.

- Implication for health policy/practice/research/medical education:

This strategy may have therapeutic value that can be applied to the treatment of infectious diseases.

- Please cite this paper as:

Chen JH, Yu YS, Liu HH, Chen XH, Xi M, Zang GQ, et al. Ubiquitin Conjugation of Hepatitis B Virus Core Antigen DNA Vaccine Leads to Enhanced Cell-Mediated Immune Response in BALB/C Mice. Hepat Mon. 2011;11(8):620-8. [DOI: 10.5812] kowsar.1735143X.689]

\footnotetext{
* Corresponding author at: Zheng-Hao Tang, Department of Infectious Diseases, Shanghai Jiaotong University Affiliated Sixth People's Hospital, 200233, Shanghai, China. Tel:+86-21643691818673, Fax:+86-2164701361.

E-mail: tzhhao@hotmail.com

DOI: 10.5812/kowsar.1735143X.689

Copyright $\odot$ 2011, BRCGL, Published by Kowsar M.P.Co. All rights reserved.
}

\section{Background}

An estimated 350 million persons worldwide are chronically infected with hepatitis B virus (HBV). HBV 
infection is a major global public health problem. Approximately600,000 deathseachyearareattributed to acuteorchronicHBVinfection(1). Although someantiviral drugs are extremely well tolerated and suppress HBV replication effectively, they rarely eliminate intranuclear viral covalently closed circular DNA (2). Therefore, it is necessary to develop an alternative, effective therapeutic approach for chronically infected patients. The antigenencoding DNA vaccine, which can effectively induce humoral and cellular immune responses, has become an attractive immunization strategy against a variety of pathogens, including HBV (3-5). A prophylactic vaccine that based on hepatitis B surface antigen is an effective way of reducing the global incidence of hepatitis (6), but it does not work therapeutically (7). HBV core antigen (HBcAg) possesses unique immunological features. Patients who successfully clear the virus usually have efficient HBcAg-specific cytotoxic T lymphocyte (CTL) responses $(8,9)$. Plasmid DNA that encodes HBcAg elicits humoral and cellular responses in many animal models $(5,10,11)$. Therapeutic DNA vaccination is a promising strategy for controlling chronic infections. However, this approach has not been as successful as initially anticipated for chronic hepatitis B. The application of DNA vaccines in humans has been limited due to their low immunogenicity (12). Many attempts have been made to enhance the potency of DNA vaccines, including codelivery of a cytokine (13) and insertion of certain sequences that enhance immune responses, such as cytokine and chemokine genes, into the vector $(14,15)$.

It is generally accepted that the primary cause of viral persistence during $\mathrm{HBV}$ infection is an inadequate antiviral response to viral antigens. Individuals who are chronically infected with HBV generally have low to undetectable CTL responses to HBV antigens. Specific $\mathrm{CD}^{+} \mathrm{T}$ cells function as CTLs, eliminating HBV (16-18). Antigen presentation to $\mathrm{CD} 8^{+} \mathrm{T}$ cells is mediated by MHC class I molecules, expressed on the surface of antigen-presenting cells. Prior to such presentation, antigens must be ubiquitinated and processed into suitable antigenic peptides by the ubiquitin-proteasome system (UPS) (19-21). The UPS is a highly selective ATPdependent proteolytic system in all eukaryotic cells that underlies antigen presentation. Ubiquitin (Ub), a highly conserved, 76-amino-acid polypeptide that is expressed in all eukaryotes, is a part of the UPS. The attachment of ubiquitin to a protein is the initial signal for its targeted degradation. When a protein is fused to ubiquitin, its degradation by the proteasome and presentation can be rapided, resulting in effectively induced immune responses. This strategy has been applied to DNA vaccines to improve immune responses by enhancing the production of antigenic peptides that are presented by MHC class I molecules (20-22).

\section{Objectives}

In the study, we constructed expression plasmids encoding mutant Ub-fused $\mathrm{HBcAg}$ and demonstrated that this fusion DNA vaccine induces humoral and cellular immune responses against HBV.

\section{Materials and Methods}

\subsection{Cell Line and Plasmids}

COS-7 cells were maintained in Dulbecco's modified Eagle's medium (Invitrogen, Gaithersburg, MD, USA), supplemented with $10 \%$ fetal bovine serum (Gibco, USA), $100 \mathrm{U} / \mathrm{ml}$ penicillin, and $100 \mu \mathrm{g} / \mathrm{ml}$ streptomycin. The $\mathrm{H}-2^{\mathrm{d}}$ mastocytoma cell line P815/c (expressing HBV c antigen) was preserved in our lab. The PADR plasmid, containing full-length HBV DNA, was provided by Prof. Yuan Wang, Shanghai Institute of Biochemistry and Cell Biology, Chinese Academy of Sciences. The eukaryotic vector pcDNA3.1 (-) was provided by Prof. Huaidong Song, Ruijin Hospital, Shanghai Jiao Tong University School of Medicine.

\section{2. Plasmid Construction and Preparation}

We used pcDNA3.1 (-) as our expression vector in eukaryotic cells to construct two plasmids: pcDNA3.1 (-) -Ub-HBcAg and pcDNA3.1 (-) -HBcAg. To construct the Ub-HBcAg fusion plasmid, the gene encoding mutant ubiquitin, whose C-terminal Gly residue was replaced with Ala, was amplified by PCR from genomic DNA from $B A L B / C$ mouse liver using primers $A$ and $B(23)$. The HBcAg gene, whose N-terminal Met residue was replaced with Arg according to the $\mathrm{N}$-end rule, was amplified from the pADR plasmid by PCR using primers $C$ and $D$. The primers are shown in Table Ub-HBcAg was spliced by overlap extension by PCR with primers A and D. The purified Ub-HBcAg fragment was inserted into EcoRI/ HindIII-digested pcDNA3.1 (-).

To construct the HBcAg DNA vaccine, the HBcAg gene was PCR-amplified from pADR using primer $E$ with a BamH I site and the antisense primer F with a Hind III site. The PCR product was digested with BamHI/Hind III and ligated into pcDNA3.1 (-). All plasmids were confirmed by restriction enzyme digestion and sequence analysis. Plasmids were grown in Escherichia coli DH5 $\alpha$ and purified using an endotoxin-free purification kit(Qiagen, Germany). The purified plasmids were resuspended in sterile PBS to $500 \mu \mathrm{g} / \mathrm{ml}$.

\section{3. In Vitro Transfection, Western Blotting}

For transfection, COS-7 cells $\left(5 \times 10^{6}\right.$ cells $\left./ \mathrm{ml}\right)$ were used to seed 6-well plates in DMEM supplemented with $10 \% \mathrm{FBS}$ and incubated for $24 \mathrm{~h}$ at $37^{\circ} \mathrm{C}$ in a humidified incubator, $5 \% \mathrm{CO}_{2}$. Cells were transiently transfected with pUb-HBcAg, pHBcAg, or pcDNA3.1(-) using Lipofectamine 2000 (Invitrogen, USA) per the manufacturer. Fortyeight hours after transfection, cells were harvested to analyze HBcAg by western blot, as described elsewhere (24). Mouse monoclonal anti-human HBcAg (Santa Cruz, USA) was used as the primary antibody, and horseradish peroxidase-conjugated goat anti-mouse IgG was used as the secondary antibody. 


\begin{tabular}{|c|c|c|}
\hline Gene Name & Nucleotide Sequence $\left(5^{\prime} \rightarrow \mathbf{3}^{\prime}\right)$ & $\begin{array}{l}\text { Restriction } \\
\text { Enzyme Site }\end{array}$ \\
\hline $\mathrm{Ub}$ & $\begin{array}{l}\text { A: CGCAGAATTC }{ }^{\mathrm{a}} \text { ATGCAGATCTTCGTGAAG } \\
\text { B: ATTCTTTATACGGGTCAATGTCTCT }{ }_{\mathrm{c}}^{\mathrm{AGC}} \text { ACCTCTCAGGCGAAGGACCAGG }\end{array}$ & EcoR I \\
\hline HBcAg & 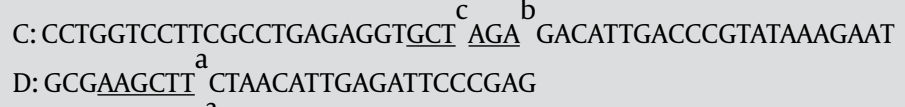 & Hind III \\
\hline HBcAg ${ }^{d}$ & $\begin{array}{l}\text { E: GATAGGATCC }{ }^{\mathrm{a}} \text { ATGGACATTGACCCGTAT } \\
\text { F: GCGAAGCTTC } \\
\text { TAACATTGAGATTCCCGAG }\end{array}$ & $\begin{array}{l}\text { BamH I } \\
\text { Hind III }\end{array}$ \\
\hline
\end{tabular}

\subsection{RT-PCR}

In order to detect expression of the DNA vaccines in our eukaryotic system, we extracted total RNA from transfected cells and analyzed it by RT-PCR. For each PCR reaction, $2 \mu \mathrm{l}$ of cDNA product of the reversetranscription reaction was used as the template. The PCR primers were as follows: Ub-HBcAg forward primer: 5'-CGCAGAATTCATGCAGATCTTCGTGAAG-3' and reverse primer:5'-GCGAAGCTTCTAACATTGAGATTCCCGAG-3'; HBcAg forward primer: 5'GATAGGATCCATGGACATTG ACCCGTAT-3' and reverse primer: 5'-GCGAAG CTTCTAACATTGAGATTCCCGAG-3', and the housekeeping gene $\beta$-actin, forward primer: $5^{\prime}$-CATCTCTTGCTCGAACA- $3^{\prime}$ and reverse primer: $5^{\prime}$-ATCATGTTTGAG ACCTTCAACA-3'. The PCR comprised the following steps: $94^{\circ} \mathrm{C}$ for $10 \mathrm{~min}$ and 32 cycles of $94^{\circ} \mathrm{C}$ for $60 \mathrm{~s}, 54^{\circ} \mathrm{C}$ for $45 \mathrm{~s}$, and $72^{\circ} \mathrm{C}$ for 120s. Finally, the reactions were incubated at $72^{\circ} \mathrm{C}$ for 10 min. The PCR products were examined by agarose gel electrophoresis.

\section{5. Mice and DNA Vaccination}

Female BALB/c mice $\left(\mathrm{H}-2^{\mathrm{d}}\right)$, 6-8 weeks old, were acquired from Shanghai Experimental Animal Center of Chinese Academy of Sciences and kept under pathogenfree conditions. The animal experiments were approved by the institutional ethical committee of the Sixth People's Hospital, Shanghai Jiaotong University. Mice were divided into 5 groups, with 8 mice in each group. To increase the efficiency of the gene transfer, the tibialis anterior muscle was injected with a total of $100 \mu \mathrm{l}$ of $0.5 \%$ aethocaine per mouse. After 24 h, $100 \mu \mathrm{g}$ pUb-HBcAg, 50 $\mu \mathrm{g}$ pUb-HBcAg, $100 \mu \mathrm{g}$ pHBcAg, $50 \mu \mathrm{g}$ pHBcAg, or $100 \mu \mathrm{g}$ pcDNA3.1(-) was injected into the same muscle in $200 \mu \mathrm{l}$ of PBS. The mice were immunized intramuscularly on Days 0,14 , and 28.

\section{6. Detection of HBcAg-Specific $\operatorname{Ig} G$}

Blood was collected on Days 0, 14, 28, and 42 after primary immunization from anesthetized mice by retro-orbital bleeding. Serum anti-HBc was measured by enzyme-linked immunosorbent assay(ELISA)(Diagnostic Reagent Center of Shanghai Municipal Infectious Diseases Hospital, Shanghai, China) in 96-well microtiter plates coated with HBcAg. Serum was serially diluted in PBS with 5\% nonfat milk (starting from 1:100) and incubated in microtiter plates for 1 hour at $37^{\circ} \mathrm{C}$. Plates were washed, and horseradish peroxidase-conjugated goat anti-mouse IgG (1:1000) was added to the wells and incubated at room temperature for 1 hour. After extensive washing, the plates were incubated at room temperature for $30 \mathrm{~min}$ with $100 \mu \mathrm{l}$ of substrate in the presence of hydrogen peroxide. The absorbance at 450 $\mathrm{nm}$ was measured. Endpoint titers were defined as the highest serum dilution that resulted in an absorbance value 2 times greater than that of negative control sera.

\subsection{Lymphocyte Preparation and Lymphocyte Proliferation Assay}

Mice were anesthetized and sacrificed 2 weeks after the last immunization, and their spleens were homogenized over 200 gauge nylon mesh. Splenocytes were collected, treated with lysis buffer to eliminate red cells, washed, and resuspended in RPMI-1640 with 10\% FBS. Lymphocytes were derived from splenocytes using nylon wool columns. Single-cell suspensions of lymphocytes $\left(5 \times 10^{5}\right.$ cells/well) were grown in 96-well plates, stimulated in vitro with $10 \mu \mathrm{g} / \mathrm{mL}$ MBP-HBcAg (purified in our laboratory) (25), and incubated at $37^{\circ} \mathrm{C}$ in $5 \% \mathrm{CO}_{2}$ for $72 \mathrm{~h}$. Cultures were incubated with $10 \mu \mathrm{L}$ CCK-8 solution (Beyotime Institute of biotechnology, Haimen, China) for $4 \mathrm{~h}$ at $37^{\circ} \mathrm{C}$. The absorbance was read at 450 nm.

\subsection{Measurement of Cytokine Production In Vitro}

To determine whether $\mathrm{T}$ helper cells were activated, we measured cytokines (IL-2, IFN- $\gamma$, IL-4, and IL-10) that reflected the Th1 and Th2 responses. Splenocytes from mice in each group were cultured in 96-well culture plates in the presence of $10 \mu \mathrm{g} / \mathrm{mL}$ MBP-HBcAg for 4 days, and the supernatants were harvested. Cytokines 
were measured using commercial ELISA kits, per the manufacturer's protocol (R\&D Systems, Minneapolis, MN, USA). Data are expressed as pg/ml.

\subsection{Measurement of Intracellular Cytokines in Proliferating T Cells}

IFN- $\gamma$ production was measured by intracellular staining and flow cytometry. Spleen cells from immunized mice were plated in 96-well plates and stimulated with MBP-HBcAg (final concentration $10 \mu \mathrm{g}$ / $\mathrm{mL}$ ) for 4 days at $37^{\circ} \mathrm{C}$ in a humidified atmosphere, $5 \% \mathrm{CO}_{2}$. Proliferating T cells were suspended in complete RPMI 1640 and stimulated for $6 \mathrm{~h}$ in the presence of $25 \mu \mathrm{g} / \mathrm{mL}$ phorbol 12-myristate 13-acetate, $1 \mu \mathrm{g} / \mathrm{mL}$ ionomycin, and $1.7 \mu \mathrm{g} / \mathrm{mL}$ monensin (Sigma, USA). After being washed with PBS, the cells were stained with FITC-conjugated anti-CD8 ${ }^{\circ} \mathrm{C} \mathrm{mAb}$ (eBioscience, USA) for 30 minutes at $4^{\circ} \mathrm{C}$, washed with PBS, fixed with $4 \%$ paraformaldehyde, and permeabilized with PBS containing 0.5\% saponin (both from BD Shanghai, China). Cells were incubated with PElabeled anti-interferon- $\gamma$ (IFN- $\gamma$ ) (eBioscience, USA) for 30 minutes at $4^{\circ} \mathrm{C}$, washed with PBS, and analyzed by flow cytometry.

\subsection{HBV-Specific CTL Activity}

Mouse splenocytes from immunized mice were stimulated in vitro for 5 days with $10 \mu \mathrm{g} / \mathrm{ml}$ MBP-HBcAg and $20 \mathrm{IU} / \mathrm{ml}$ recombinant murine IL-2. The restimulated splenocytes $\left(5 \times 10^{6} / \mathrm{mL}\right)$ were used as effectors. The P815/c cell line was used as the target cell. P815/c cells were seeded at $5 \times 10^{4}$ cells/well in 96-well plates and cultured for $12 \mathrm{~h}$. Effector cells were incubated with P815/c at various effector/target $(\mathrm{E} / \mathrm{T})$ ratios (12.5:1, 25:1, or 50:1) for $4 \mathrm{~h}$. The HBcAg-specific CTL activity was measured by lactate dehydrogenase (LDH) release assay per the manufacturer's instructions using the CytoTox 96® Non-Radioactive Cytotoxicity Assay (Promega, USA). The absorbance values from supernatants were recorded at OD $450 \mathrm{~nm}$. The percentage of cytotoxicity was calculated as follows: [(Experimental release - Effector spontaneous release - Target spontaneous release)/(Target maximum release - Target spontaneous release) $] \times 100 \%$.

\subsection{Statistical Analysis}

Results were expressed as mean \pm SD. The statistical significance of differences between 2 groups was determined by student's t-test, and differences between 2 or more groups were analyzed by one-factor analysis of variance (ANOVA). Data were considered statistically significant at $P<0.05$.

\section{Results}

\subsection{Construction and Expression of DNA Vaccines}

The ubiquitin gene was PCR-amplified from genomic DNA from the liver of BALB/C mice, producing a band of $552 \mathrm{bp}$; splicing of the ubiquitin and HBcAg genes by overlap extension generated a product of $780 \mathrm{bp}$. The fragment was purified and cloned into pcDNA3.1 (-). Recombinant plasmids were confirmed by restriction enzyme digestion (Figure 1) and sequencing. The sequences of the DNA vaccines were confirmed by sequencing. The expression of HBcAg or the fusion protein (Ub-HBcAg) was detected by RT-PCR and western blot $48 \mathrm{~h}$ after the plasmids were transfected into COS-7 cells. By RT-PCR of pHBcAg transfectants, we amplified about a product of approximately $550 \mathrm{bp}$, consistent with the HBcAg gene (552 bp), and a 780-bp product from cells that were transfected with pUb-HBcAg (Figure 2A). No similarly sized bands were seen in the negative control RNA. By western blot, 21-kD proteins were expressed in the lysates of COS-7 cells that were transfected with pUb-HbcAg and pHBcAg. pcDNA3.1 (-) transfectants did not express any such proteins. (Figure $2 B$ ). These results confirm that HBcAg and Ub-HBcAg can be expressed in a eukaryotic system.

\section{2. Production of HBc-Apecific Antibody by DNA Vaccination}

The anti-HBc response that was induced by the vaccines was evaluated by ELISA. At 14 days after priming, there was no significant difference in the endpoint titers of anti-HBc between groups. As shown in Figure 3 , robust anti-HBc IgG responses were elicited in mice that were immunized with $100 \mu \mathrm{g}$ pUb-HBcAg; the endpoint titers of anti-HBc was 1:72,900, $28 \mathrm{~d}$ after the first immunization. The antibody titers at 28 days were significantly higher in the group that was immunized with $100 \mu \mathrm{g}$ pUb-HBcAg versus $100 \mu \mathrm{g}$ pHBcAg $(P=0.001)$. At 42 days after priming, $100 \mu \mathrm{g}$ pUb-HBcAg significantly enhanced antibody responses compared with pHBcAg at the same dose $(P<0.01)$. The endpoint titers of anti$\mathrm{HBc}$ in mice that were immunized with pUb-HBcAg 100 $\mu \mathrm{g}$ peaked at 1:656,100 on Day 42. No anti-HBcAg was detected in the serum of control mice that were injected with pcDNA3.1 (-). These results indicate that pUb-HBcAg enhances humoral immune responses.

\subsection{Lymphocyte Proliferation and Cytokine Production by Splenocytes from Vaccinated Mice}

After $72 \mathrm{~h}$, lymphocyte proliferation was measured by CCK-8 assay. Compared with the $100 \mu \mathrm{g}$ pHBcAg and 50 $\mu \mathrm{g}$ pHBcAg groups, pUb-HBcAg induced significantly greater lymphocyte proliferation $(P<0.01)$. pHBcAg $(100$ $\mu \mathrm{g})$ effected greater lymphocyte proliferation than 50 $\mu \mathrm{g}$ pHBcAg $(P<0.05)$. As seen in Figure 4 , the pUb-HBcAg $100 \mu \mathrm{g}$ group experienced the greatest lymphocyte proliferation, indicating that vaccination with Ub-fused HBcAg induces the proliferation of specific lymphocytes. Th1 (IFN- $\gamma$, IL-2) and Th2 cytokines (IL-4, IL-10) were measured in the supernatants of splenocytes ELISA. pUb$\mathrm{HBCAg} 100 \mu \mathrm{g}$ and pUb-HBcAg $50 \mu \mathrm{g}$ induced higher levels 
Figure 1. Electrophoresis of pUb-HBcAg Digested by EcoR I and Hind III

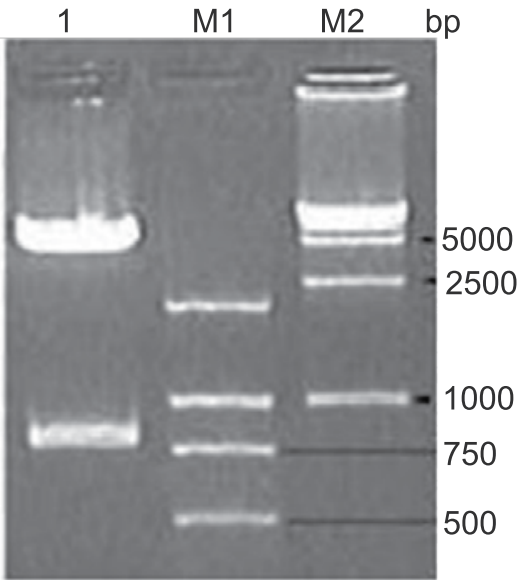

Lane 1:pUb-HBcAg Digested by EcoR I and Hind III; Lane M1:DNAMarker 2000; Lane M2:DNA Marker 15000.

Figure 3. HBcAg-Specific IgG Titer in the Sera of Mice Immunized with Various Formulations

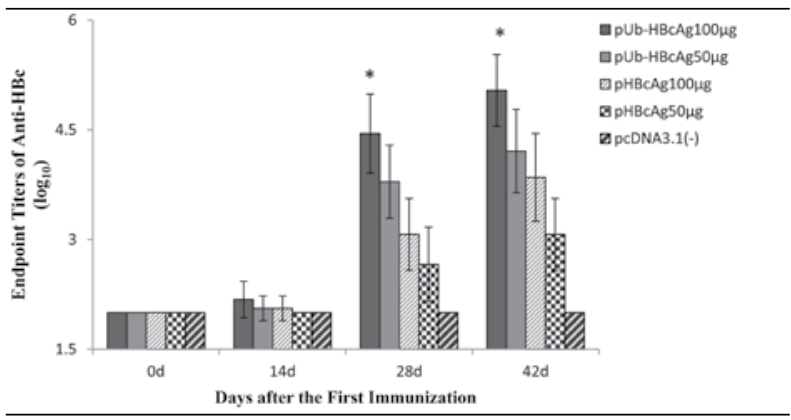

The initial dilution of each serum from immunized mouse was 1:100 and followed with serial of three-fold dilution for anti-HBc IgG detection. Data is from one representative experiment of three performed and is presented as

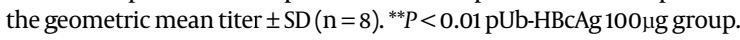

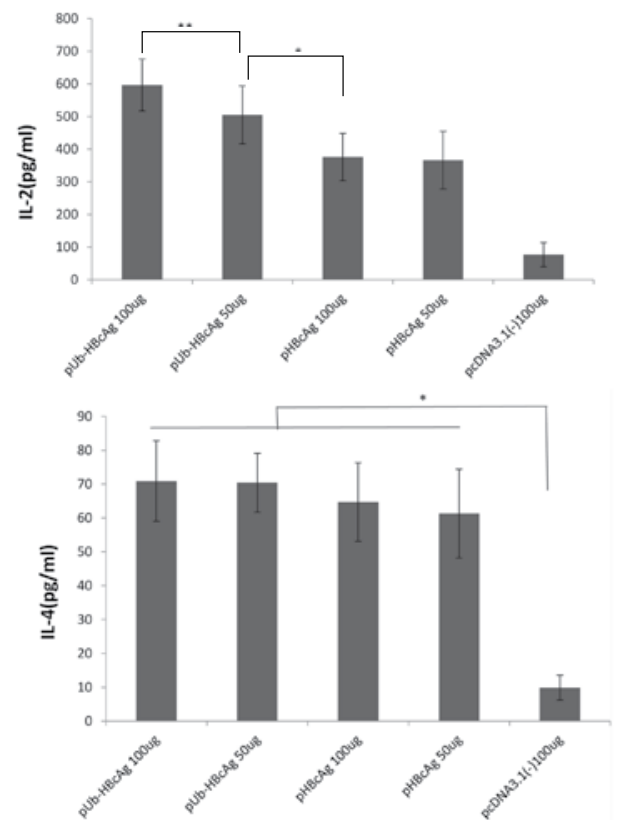

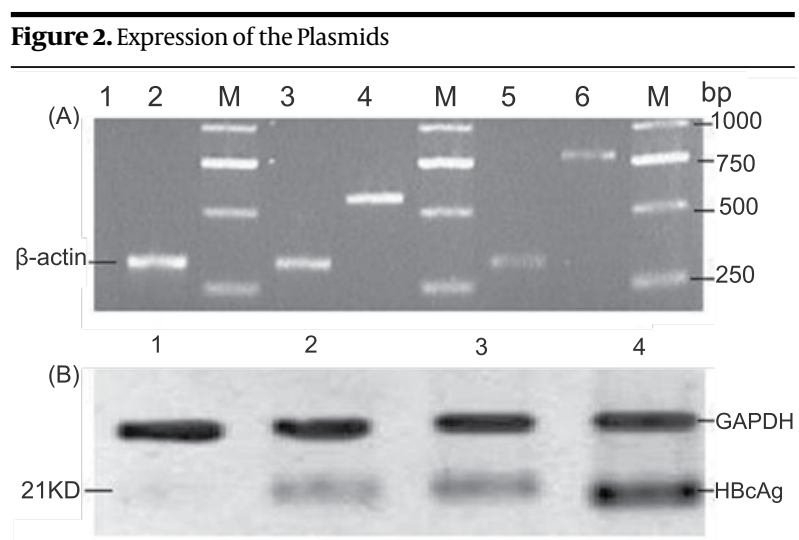

(A) Gene expression of HBcAg and Ub-HBcAg, Lane M: DNA marker 2000; Lane 1: blank plasmid pcDNA3.1 (-) transfecting COS-7 cells; Lane 2,3,5: $\beta$-actin; Lane 4: plasmid pHBcAg transfecting COS-7 cells; Lane 6: plasmid pUb-HBcAg transfecting COS-7 cells. (B) Protein expression of HBcAg (about $21 \mathrm{kDa}$ ), Lane 1: COS-7 cells transfected with pcDNA3.1 (-); Lane 2 and Lane 3 are the same sample: COS-7 cells transfected with pUb-HbcAg; Lane 4: COS-7 cells transfected with pHBcAg

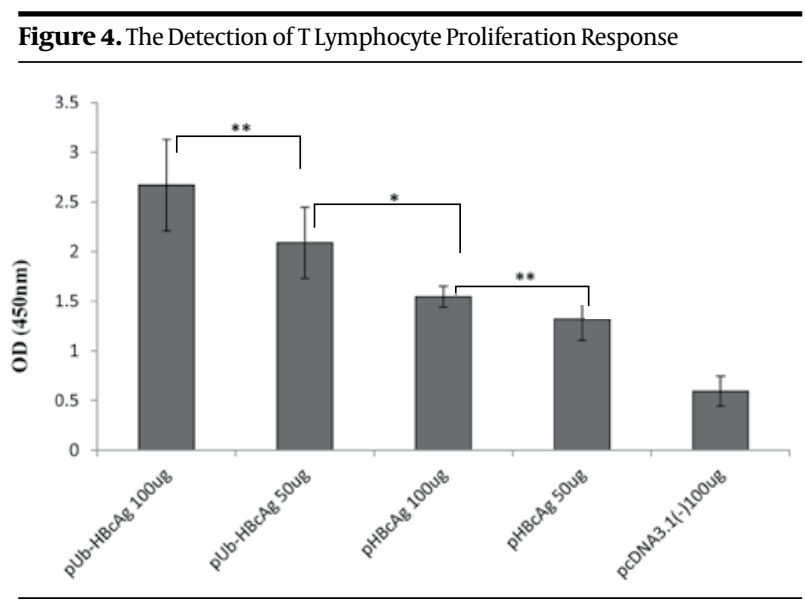

Data shown represent the mean and SD. ${ }^{* *} P<0.05,{ }^{*} P<0.01$
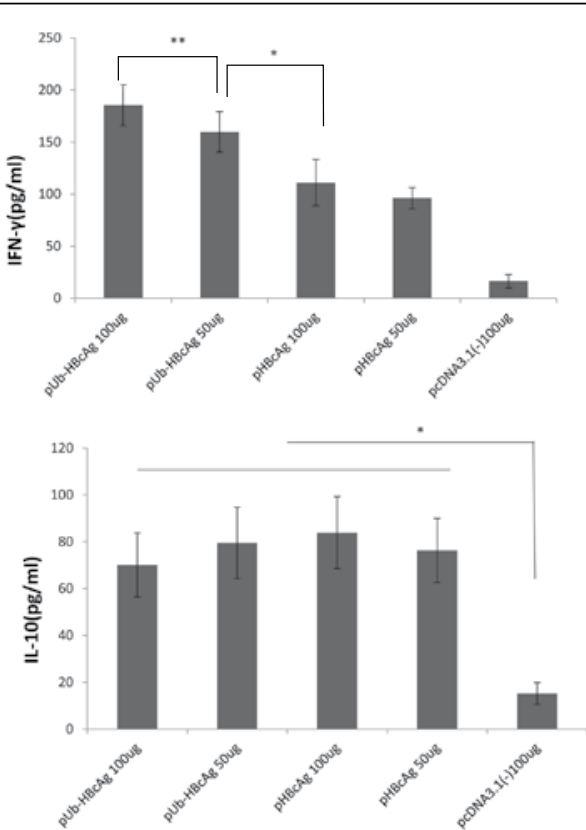

Figure 5. Cytokines Production in the Supernatant of Splenocytes Harvested from Immunized Mice after in Vitro Re-stimulation. Data Represent the Means \pm $\mathrm{SD}(\mathrm{n}=8)$. 


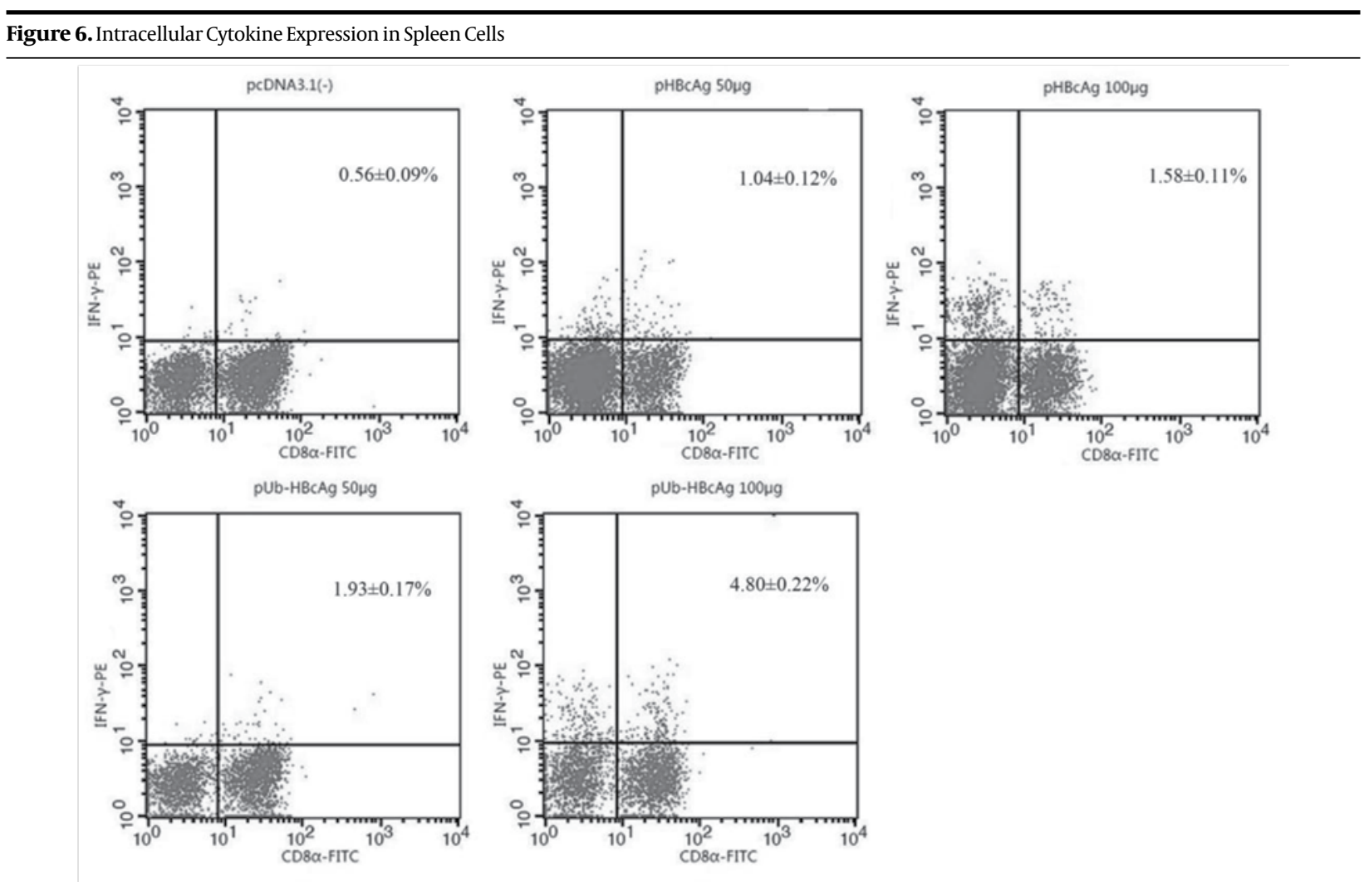

The whole cell population was doubly stained with fluorescent material labeled using FITC-CD8 $\alpha$ and PE-IFN- $\gamma$ antibodies. The doubly stained cells were counted and analyzed by flow cytometry. The data are the mean \pm SD from eight mice per group.

$\overline{\text { Figure 7.HBcAg-Specific CTLActivityWasMeasured byLactateDehydrogenase }}$ Release Assay

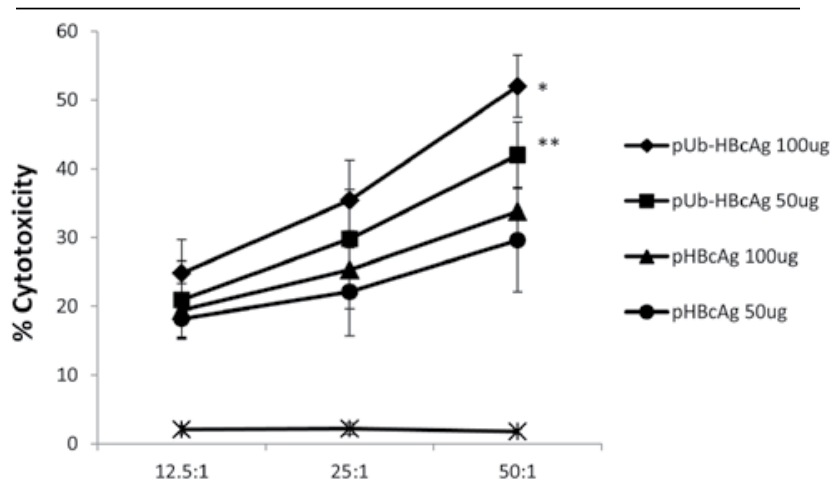

$\overline{\text { CTL activity is indicated as the mean percentage of specific lysis } \pm \text { SD at }}$ different effector:target (E:T) ratios. Experiments were repeated three times with similar results. ${ }^{*} P<0.01$ pUb-HBcAg $100 \mu$ g group $v s$. pUb-HBcAg $50 \mu \mathrm{g}$

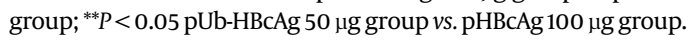

of IL-2 than pHBcAg. As shown in Figure 5, IFN- $\gamma$ levels in the pUb-HBcAg $100 \mu \mathrm{g}$ group were also much higher compared with the other plasmids. Compared with pUbHBcAg $50 \mu \mathrm{g}(159.8 \pm 19.55 \mathrm{pg} / \mathrm{mL})$, pUb-HBcAg $100 \mu \mathrm{g}$ elicited significantly higher production of IFN- $\gamma$ (185.4 \pm $19.63 \mathrm{pg} / \mathrm{mL})(P<0.05)$. Mice that were immunized with pUb-HBcAg and pHBcAg produced more Th2 cytokines (IL-4, IL-10) than the control group $(P<0.01)$. However, Th2 cytokine levels did not differ significantly between the pUb-HBcAg and pHBcAg groups.

\section{4. Specific CD8 ${ }^{+}$T Cells Response}

Mice from each group were sacrificed, and splenocytes were isolated after the last immunization and restimulated in vitro. Cells were doubly stained with FITC-CD $8 \alpha$ and PE-IFN- $\gamma$ antibodies, counted, and analyzed by flow cytometry. IFN- $\gamma$ expression was higher with pUb-HBcAg than in the pHBcAg samples. The percentage of IFN- $\gamma$-positive T cells in the CTL population was significantly higher with $100 \mu \mathrm{g}$ pUb-HBcAg versus pHBcAg. (Figure 6). Thus, pUb-HBcAg was effective in generating CTLs.

\subsection{HBcAg-Specific CTL Response}

To evaluate the specific cytotoxicity of T lymphocytes in the various groups of immunized mice, the $\mathrm{LDH}$ relaxation index was measured. Splenocytes from immunized mice were restimulated in vitro with MBPHBcAg and rmIL-2. HBcAg-specific CTL activity at various effector/target ratios is shown in (Figure 7). T lymphocytes from mice that were vaccinated with pUb-HBcAg 100 $\mu \mathrm{g}$ killed $52.0 \pm 4.5 \%$ of target cells at an effector: target ratio of 50:1, significantly higher than with pUb-HBcAg $50 \mu \mathrm{g}(42.0 \pm 4.8 \%)(P<0.01)$. pUb-HBcAg $50 \mu \mathrm{g}$ elicited a greater CTL response, compared with pHBcAg $100 \mu \mathrm{g}$, at an effector:target ratio of 50:1 $(P<0.05)$. At 25:1, the 
CTL response against pUb-HBcAg $100 \mu \mathrm{g}$ was higher versus the other groups $(P<0.05)$. These results indicate that mice that are immunized with pUb-HBcAg develop strong, specific CTL responses.

\section{Discussion}

Immune responses play a significant role in the clearance of hepatitis B virus. Protective immunity against HBV depends on both the humoral and cellular immune responses. Vigorous polyclonal and multispecific immune responses to $\mathrm{HBV}$ have been detected in acute infections. In contrast, defective CTLand Th cell responses have been observed in patients who are chronically infected HBV (16). It has been reported that specific cellular immunity against $\mathrm{HBV}$ is a key factor in the control of $\mathrm{HBV}$ infection $(26,27)$. One promising method is the use of DNA vaccines, which have the ability to induce antigen-specific cellular immune responses (28-30). A DNA vaccine harbors DNA-encoded antigens that are subjected to UPS degradation, resulting in peptide fragments that can be presented by MHC class I antigens to $\mathrm{CD}^{+} \mathrm{T}$ cells, mimicking a microbial infection. Although a DNA vaccine can break immune tolerance, it is unable to completely eliminate the viral particle load. In order to improve the efficacy of DNA vaccines, some strategies have been explored $(31,32)$. In this study, we aimed to develop a more powerful HBV DNA vaccine by constructing a plasmid that contains both the Ub and HBcAg genes.

Ubiquitin is a small, highly conserved regulatory protein that is ubiquitous in eukaryotes. The most significant function of ubiquitin in proteolysis is its role as a signal for a target protein to be recognized and degraded in the proteasome (33). T cell receptors recognize short peptides that are presented by MHC class I molecules on antigen-presenting cells $(34,35)$. Higher rates of intracellular antigen traffic should increase the number and variety of peptides that are available for MHC class I binding, increasing the immune response to the expressed antigen. A very effective way of improving antigenic presentation by DNA vaccination is to fuse the Ub gene to the target antigen. A direct correlation between rapid ubiquitin-mediated processing of antigens and enhanced cell-mediated immune responses has been established (36). It has also become apparent that ubiquitylation is a reversible reaction, in which ubiquitin chains are conjugated and deconjugated by ubiquitylating and deubiquitylating enzymes, respectively (37). To prevent cleavage of the fusion gene by deubiquitination enzymes, we constructed an expression vector encoding HBcAg fused to Ub, in which the C-terminal glycine of Ub was replaced with alanine. In addition, the $\mathrm{N}$-terminal Met residue of HBcAg was replaced by Arg. Thus, the fusion protein could be quickly recognized by the UPS, enhancing HBcAg degradation.

Previous attempts of tagging antigens with ubiquitin have placed Arg at the N-terminus to target them for rapid processing (38-40). By RT-PCR, the cassettes of the Ub-HBcAg constructs were efficiently transcribed, and by western blot, HBcAg was translated from the expression vectors. As expected, ubiquitin-fused HBcAg converted into an excellent substrate for the UPS. We found that COS-7 cells that transfected with pUb-HBcAg expressed low levels of protein.

The function of our DNA vaccines was compared by measuring their effects on immune responses in $\mathrm{BALB} / \mathrm{c}$ mice. We found that pUb-HBcAg enhanced the recruitment and activation of $\mathrm{T}$ cells and increased the number of antigen-specific $\mathrm{CD} 8^{+} / \mathrm{IFN}-\gamma^{+} \mathrm{T}$ cells. In this study, we noted enhanced lymphocyte proliferation in mice that were vaccinated with ubiquitinated $\mathrm{HBcAg}$ compared with nonubiquitinated HBcAg. This result shows that ubiquitin conjugation increased the cellmediated immune response.

Antigen-specific $\mathrm{CD}^{+} \mathrm{T}$ cells play a vital role in the control of viral infections. They can remove infected target cells through cytotoxic or noncytotoxic activities, such as the production of IFN- $\gamma$ and other Th1 cytokines (41). It is believed that Th1 cells primarily secrete IL-2 and IFN- $\gamma$, whereas Th2 cells secrete the type II cytokines IL-4 and IL-10. We measured IL-2, IFN- $\gamma$, IL-10, and IL-4 in the spleens of mice that were immunized with our vaccines. Mice that were given ubiquitinated HBcAg expressed higher levels of IL-2 and IFN- $\gamma$ compared versus nonubiquitinated HBcAg. T cell-derived cytokines can enhance antigen-specific $\mathrm{T}$ cell populations. In mice that were immunized by pUb-HBcAg, the number of HBcAg-specific CD8 $8^{+} / \mathrm{IFN}-\gamma^{+} \mathrm{T}$ cells in spleen was higher than in pHBcAg recipients, indicating that the immune responses are type 1 rather than type 2 . The Th1 cell has been reported to correlate with the induction of CTL activity, which is beneficial for viral or tumor eradication $(42,43)$. Inadequate endogenous antigen presentation by MHC class I molecules to $\mathrm{CD}^{+} \mathrm{T}$ cells is one of the reasons why our immune system fails to eliminate pathogens. Patients with $\mathrm{CHB}$ or therapeutic failure present with deficient Th1 immunity, associated with inefficient CD8 ${ }^{+}$ $\mathrm{T}$ cell cytotoxicity. In our study, the enhancement of antigen presentation increased the number of antigenspecific $\mathrm{CD}^{+} / \mathrm{IFN}-\gamma^{+} \mathrm{T}$ cells in pUb-HBcAg-immunized mice. Ubiquitin-fused HBcAg is rapidly degraded by the proteasome, resulting in efficient production of a variety of peptides, including many CTL epitopes, that might be presented by many types of MHC class I molecules.

As shown in Figure 7, mice that were immunized with pUb-HBcAg $100 \mu \mathrm{g}$ showed substantial cytotoxic activity against target cells, while those immunized with pUbHBcAg $50 \mu$ g or pHBcAg showed remarkably enhanced cytotoxicity. We have clearly shown that immunization a ubiquitin-fuse HBcAg DNA vaccine induces robust HBcAg-specific immune responses in BALB/c mice, consistent with previous reports $(44,45)$. pUb-HBcAg can not only induce cell-mediated immune responses but also enhance humoral immune responses. We observed very high levels of anti-HBc IgG in mice that 
were immunized with pUb-HBcAg $100 \mu$ g compared with other plasmids. These results suggest that pUb-HBcAg elicits strong, specific anti-HBc humoral responses in BALB/c mice after DNA vaccination. However, in Vidalin's study (46), when ubiquitin was fused to hepatitis $C$ virus core antigen, the antibody response was undetectable compared with the nonfusion vaccine. This difference in result might be attributed to the disparate antigenicities of the proteins.

Both pUb-HBcAg and pHBcAg induced humoral and cellular immune responses in normal mice, but pUbHBcAg provoked a more robust response, suggesting that when the Ub gene is fused to the HBcAg gene, the efficacy of the DNA vaccine increases. Therefore, this novel strategy may have therapeutic value in infectious diseases and cancer.

\section{Acknowledgments}

This work was supported by grants from the Natural Science Fund of Shanghai (number 11ZR1427100).

\section{Financial Disclosures}

None declared.

\section{Funding/Support}

This work was supported by grants from the Natural Science Fund of Shanghai (number 11ZR1427100).

\section{References}

1. Te HS, Jensen DM. Epidemiology of hepatitis B and C viruses: a global overview. Clin Liver Dis. 2010;14(1):1-21, vii.

2. Cova L. DNA vaccine: a promising new approach for chronic hepatitis B therapy. Future Virol. 2007;2(5):421-4.

3. Zucchelli S, Capone S, Fattori E, Folgori A, Di Marco A, Casimiro D, et al. Enhancing B- and T-cell immune response to a hepatitis $C$ virus E2 DNA vaccine by intramuscular electrical gene transfer. $J$ Virol. 2000;74(24):11598-607.

4. Schneider J, Gilbert SC, Blanchard TJ, Hanke T, Robson KJ, Hannan $\mathrm{CM}$, et al. Enhanced immunogenicity for CD8+ T cell induction and complete protective efficacy of malaria DNAvaccination by boosting with modified vaccinia virus Ankara. Nat Med. 1998;4(4):397-402.

5. Huang ZH, Zhuang H, Lu S, Guo RH, Xu GM, Cai J, et al. Humoral and cellular immunogenecity of DNA vaccine based on hepatitis B core gene in rhesus monkeys. World J Gastroenterol. 2001;7(1):102-6.

6. Yazdanpanah B, Safari M,Yazdanpanah S. Persistence of HBVVaccine's Protection and Response to Hepatitis B Booster Immunization in 5-to 7-Year-Old Children in the Kohgiloyeh and Boyerahmad Province, Iran. Hepat Mon. 2010;10(1):17-21.

7. Moingeon P, Almond J, de Wilde M. Therapeutic vaccines against infectious diseases. Curr Opin Microbiol. 2003;6(5):462-71.

8. Cao T, Lazdina U, Desombere I, Vanlandschoot P, Milich DR, Sallberg $M$, et al. Hepatitis $B$ virus core antigen binds and activates naive human B cells in vivo: studies with a human PBL-NOD/SCID mouse model.J Virol. 2001;75(14):6359-66.

9. Tang TJ, de Man RA, Kusters JG, Kwekkeboom J, Hop WC, van der Molen RG, et al. Intrahepatic CD8 T-lymphocytes and HBV core expression in relation to response to antiviral therapy for chronic hepatitis B patients. J Med Virol. 2004;72(2):215-22.

10. Lu M, Hilken G, Kruppenbacher J, Kemper T, Schirmbeck R, Reimann $\mathrm{J}$, et al. Immunization of woodchucks with plasmids expressing woodchuck hepatitis virus (WHV) core antigen and surface antigen suppresses WHV infection. J Virol.1999;73(1):281-9.

11. Kuhober A, Pudollek HP, Reifenberg K, Chisari FV, Schlicht HJ, Reimann J, et al. DNA immunization induces antibody and cytotoxic $\mathrm{T}$ cell responses to hepatitis B core antigen in $\mathrm{H}-2 \mathrm{~b}$ mice. J Immunol. 1996;156(10):3687-95.

12. Miche ML, Deng Q, Mancini-Bourgine M. Therapeutic vaccines and immune-based therapies for the treatment of chronic hepatitis $B$ : perspectives and challenges. J Hepatol. 2011;54(6):1286-96.

13. Dahmen A, Herzog-Hauff S, Bocher WO, Galle PR, Lohr HF. Clinical and immunological efficacy of intradermal vaccine plus lamivudine with or without interleukin-2 in patients with chronic hepatitis B.J Med Virol. 2002;66(4):452-60.

14. Qing $\mathrm{Y}$, Chen $\mathrm{M}$, Zhao J, $\mathrm{Hu} \mathrm{H}, \mathrm{Xu} \mathrm{H}$, Ling N, et al. Construction of an HBV DNA vaccine by fusion of the GM-CSF gene to the HBV-S gene and examination of its immune effects in normal and HBVtransgenic mice. Vaccine. 2010;28(26):4301-7.

15. Zhou C, Peng G, Jin X, Tang J, Chen Z. Vaccination with a fusion DNA vaccine encoding hepatitis $B$ surface antigen fused to the extracellular domain of CTLA4 enhances HBV-specific immune responses in mice: implication of its potential use as a therapeutic vaccine. Clin Immunol. 2010;137(2):190-8.

16. Jung MC, Pape GR. Immunology of hepatitis B infection. Lancet Infect Dis. 2002;2(1):43-50.

17. Guidotti LG, Ishikawa T, Hobbs MV, Matzke B, Schreiber R, Chisari FV. Intracellular inactivation of the hepatitis B virus by cytotoxic T lymphocytes. Immunity. 1996;4(1):25-36.

18. Thimme R, Wieland S, Steiger C, Ghrayeb J, Reimann KA, Purcell RH, et al. CD8(+)T cells mediate viral clearance and disease pathogenesis during acute hepatitis B virus infection. J Virol. 2003;77(1):68-76.

19. Rock KL, York IA, Saric T, Goldberg AL. Protein degradation and the generation of MHC class I-presented peptides. Adv Immunol. 2002;80:1-70.

20. Zhang M, Obata C, Hisaeda H, Ishii K, Murata S, Chiba T, et al. A novel DNA vaccine based on ubiquitin-proteasome pathway targeting 'self'-antigens expressed in melanoma/melanocyte. Gene Ther. 2005;12(13):1049-57.

21. Kloetzel PM, Ossendorp F. Proteasome and peptidase function in MHC-class-I-mediated antigen presentation. Curr Opin Immunol. 2004;16(1):76-81.

22. Wang QM, Kang L, Wang XH. Improved cellular immune response elicited by a ubiquitin-fused ESAT-6 DNA vaccine against Mycobacterium tuberculosis. Microbiol Immunol. 2009;53(7):384-90.

23. Woo SK, Lee JI, Park IK, Yoo YJ, Cho CM, Kang MS, et al. Multiple ubiquitin C-terminal hydrolases from chick skeletal muscle. J Biol Chem. 1995;270(32):18766-73.

24. Yan J, Liu X, Wang Y, Jiang X, Liu H, Wang M, et al. Enhancing the potency of HBV DNA vaccines using fusion genes of HBV-specific antigens and the N-terminal fragment of gp96. J Gene Med. 2007;9(2):107-21.

25. Chen X, Yu Y, Pan Q, Tang Z, Han J, Zang G. Enhancement of cytotoxic $T$ lymphocyte activity by dendritic cells loaded with Tat-protein transduction domain-fused hepatitis B virus core antigen. Acta Biochim Biophys Sin (Shanghai). 2008;40(12):996-1004.

26. Rehermann B, Lau D, Hoofnagle JH, Chisari FV. Cytotoxic T lymphocyte responsiveness after resolution of chronic hepatitis $B$ virus infection. J Clin Invest. 1996;97(7):1655-65.

27. Bertoletti A, Gehring AJ. The immune response during hepatitis B virus infection.J Gen Virol. 2006;87(Pt 6):1439-49.

28. Geissler M, Gesien A, Tokushige K, Wands JR. Enhancement of cellular and humoral immune responses to hepatitis $C$ virus core protein using DNA-based vaccines augmented with cytokine-expressing plasmids. JImmunol.1997;158(3):1231-7.

29. Syrengelas AD, Levy R. DNA vaccination against the idiotype of a murine B cell lymphoma: mechanism of tumor protection. $J$ Immunol.1999;162(8):4790-5.

30. Sijts A, Zaiss D, Kloetzel PM. The role of the ubiquitin-proteasome pathway in MHC class I antigen processing: implications for vaccine design. Curr Mol Med. 2001;1(6):665-76.

31. Chen X, Lai J, Pan Q, Tang Z, Yu Y, Zang G. The delivery of HBcAg via Tat-PTD enhances specific immune response and inhibits Hepatitis $\mathrm{B}$ virus replication in transgenic mice. Vaccine. 2010;28(23):3913-9.

32. Wang J, Dong S, Liu C, Wang W, Sun S, Gu J, et al. beta-Glucan oligosaccharide enhances CD8(+) T cells immune response induced by a DNA vaccine encoding hepatitis B virus core antigen.J Biomed 
Biotechnol. 2010; [Epub ahead of print].

33. Gao G, Luo H. The ubiquitin-proteasome pathway in viral infections. Can J Physiol Pharmacol. 2006;84(1):5-14.

34. Bhoj VG, Chen ZJ. Ubiquitylation in innate and adaptive immunity. Nature. 2009;458(7237):430-7.

35. Kessler BM, Glas R, Ploegh HL. MHC class I antigen processing regulated by cytosolic proteolysis--short cuts that alter peptide generation. Mol Immunol. 2002;39(3-4):171-9.

36. Wu Y, Kipps TJ. Deoxyribonucleic acid vaccines encoding antigens with rapid proteasome-dependent degradation are highly efficient inducers of cytolytic T lymphocytes. J Immunol. 1997;159(12):603743.

37. Sun SC. Deubiquitylation and regulation of the immune response. Nat Rev Immunol. 2008;8(7):501-11.

38. Mogk A, Schmidt R, Bukau B. The N-end rule pathway for regulated proteolysis: prokaryotic and eukaryotic strategies. Trends Cell Biol. 2007;17(4):165-72.

39. Delogu G, Howard A, Collins FM, Morris SL. DNA vaccination against tuberculosis: expression of a ubiquitin-conjugated tuberculosis protein enhances antimycobacterial immunity. Infect Immun. 2000;68(6):3097-102.

40. Rodriguez F, Zhang J, Whitton JL. DNA immunization: ubiquitination of a viral protein enhances cytotoxic T-lymphocyte induction and antiviral protection but abrogates antibody induction. $J$ Virol. 1997;71(11):8497-503.

41. Rehermann B, Nascimbeni M. Immunology of hepatitis B virus and hepatitis C virus infection. Nat Rev Immunol. 2005;5(3):215-29.

42. Chamoto K, Kosaka A, Tsuji T, Matsuzaki J, Sato T, Takeshima T, et al. Critical role of the Th1/Tc1 circuit for the generation of tumorspecific CTL during tumor eradication in vivo by Th1-cell therapy. Cancer Sci. 2003;94(10):924-8.

43. Milich DR, Schodel F, Hughes JL, Jones JE, Peterson DL. The hepatitis $B$ virus core and e antigens elicit different Th cell subsets: antigen structure can affect Th cell phenotype. J Virol. 1997;71(3):2192-201.

44. Brandsma JL, Shlyankevich M, Zelterman D, Su Y. Therapeutic vaccination of rabbits with a ubiquitin-fused papillomavirus E1, E2, E6 and E7 DNA vaccine. Vaccine. 2007;25(33):6158-63.

45. Sharma A, Madhubala R. Ubiquitin conjugation of open reading frame F DNA vaccine leads to enhanced cell-mediated immune response and induces protection against both antimonysusceptible and -resistant strains of Leishmania donovani. Immunol. 2009;183(12):7719-31.

46. Vidalin O, Tanaka E, Spengler U, Trepo C, Inchauspe G. Targeting of hepatitis $\mathrm{C}$ virus core protein for MHC I or MHC II presentation does not enhance induction of immune responses to DNA vaccination. DNA Cell Biol.1999;18(8):611-21. 